\title{
Pricing Strategies of Audiovisual Content Products in the New Media Platform
}

\author{
Wei Lu and Ye Cao \\ The Communication University of China, Beijing, China
}

\begin{abstract}
Based on the analysis of the two-sided market characteristics of the new media audio-visual sites and the network externality of the platform, the pricing model is used to determine the factors that affect the pricing of the new media platform operators to two-sided users under the monopolistic or competitive two-sided market conditions, namely, the externality effect of the cross network and the degree of platform differentiation. Reasonable suggestions and prospects for the pricing of new media audio-visual content products are put forward.
\end{abstract}

Keywords—new media; audiovisual content products; two-sided market; pricing strategy

\section{INTRODUCTION}

In recent years, with the expansion of the influence of new media on the life of our economy and the livelihood of the people, video content products based on the internet are becoming more and more popular. The pricing of video content providers and users by the new media platform is the key problem of platform operation and management. The rationality and scientificity of the pricing methods and strategies need to be improved urgently.

\section{ANALYSIS OF BUSINESS MODE OF AUDIOVISUAL CONTENT WEBSITE}

\section{A. Content Product Sale}

The profit of content products of new media means that new media can make profits through paid audiovisual products. The new media platform stimulates audience payment (such as recharging members) by providing value-added services for various audiovisual content products to achieve profitability.

\section{B. Advertising Resources Sale}

The new media is the same as the traditional media - by providing content products to gather a large number of audience resources and in order to attract advertisers to launch ads on the platform.

In addition, new media platforms can acquire various benefits through various innovative ways, including copyright sales, crowd-funding, multi screen interaction and e-commerce.

At present, China's audiovisual content website still takes user fees and advertising revenue as the main form.

\section{ANALYSIS OF NEW MEDIA TWO-SIDED MARKET}

The new media platform belongs to the audience created two-sided market, also known as the advertising media twosided market.
The two-sided market is the market where the supplier and the service provider and the recipient trade through an intermediary platform. In such a market, the utility of a group of users will be affected by the users of another group. Therefore, the pricing of a user on one side depends not only on the cost of the user's needs and services, but also on the needs of the other side and the cost of the user on the other side of the service. That is to say, the price of a user depends on the elasticity of demand and marginal cost of the two parties.

The two-sided market has the following characteristics: (1) the demand complementarities of the two-sided market, the existence of a class of platform operators in the market by providing products or services to the users on both sides to make them trade, and the value of the platform operators depends on whether the users on both sides have a demand for the products or services on the platform; (2) two-sided markets. The cross network externality means that the utility of the platform operators is affected by the user size on the other side, and Van Alstyne (2005) calls the cross network external effect as the externality of the cross market network. If the utility of the user on the other side is increasing with the expansion of the number of users on the other side, the user is subjected to the external effect of the cross network on the other side of the user and vice versa; (3) the price structure of the two-sided market is not neutral, which means that the price structure of the platform operators to their two-sided users is often not. Symmetric, that is, relative to the general price level, the value structure has a greater impact on the two-sided user access behavior.

\section{Competitive NeW Media Platform Pricing Model}

\section{A. Basic Assumptions}

The model assumes that there are two new media platforms competing in the market, and the linear length of the market is 1 unit. The users of $c_{i}$ units and the advertisers of $a_{i}$ units are involved in the platform transaction $(i=1,2)$, and the two-sided users are evenly distributed in the market and the sum total is 1 units, namely, $c_{1}+c_{2}=1, a_{1}+a_{2}=1$. This article assumes that the user only chooses a new media platform for the transaction; namely, single ownership (single-homing), users choose a number of new media platforms to deal with them. $p_{C_{i}}$ and $p_{A_{i}}$, respectively, represent the price of unit products or services charged by the new media platform to their consumers and advertisers, and the utility function of the two-sided users is: 


$$
\left\{\begin{array}{l}
u_{C_{i}}=\alpha_{C} \cdot a_{i}-p_{C_{i}} \\
u_{A_{i}}=\alpha_{A} \cdot c_{i}-p_{A_{i}}
\end{array}\right.
$$

$\lambda_{1}$ and $\lambda_{2}$ represent the unit distance cost of two-sided users arriving at any competing platform, that is, the differentiation coefficient of the two new media platforms involved in competition.

This paper assumes that a consumer (or advertiser) in $t_{0}$ on the market has the same effect as platform 1 or 2 in the transaction:

$$
\begin{gathered}
\alpha_{C} \cdot a_{i}-p_{C_{i}}-\lambda_{1} \cdot t_{0}=\alpha_{C} \cdot a_{j}-p_{C_{j}}-\lambda_{1} \cdot\left(1-t_{0}\right)(i, j= \\
1,2)(i \neq j)
\end{gathered}
$$

Solution:

$$
t_{0}=\frac{\alpha_{C} \cdot a_{i}-p_{C_{i}}-\left(\alpha_{C} \cdot a_{j}-p_{C_{j}}\right)+\lambda_{1}}{2 \lambda_{1}}
$$

According to the Hotelling model while two-sided users are evenly distributed in the market:

$$
\begin{gathered}
c_{i}=P\left(t \leq t_{0}\right)=t_{0} \Rightarrow \\
c_{i}=t_{0}=\frac{\alpha_{C} \cdot a_{i}-p_{C_{i}}-\left(\alpha_{C} \cdot a_{j}-p_{C_{j}}\right)+\lambda_{1}}{2 \lambda_{1}}= \\
\frac{\alpha_{C}\left(2 a_{i}-1\right)-\left(p_{C_{i}}-p_{C_{j}}\right)+\lambda_{1}}{2 \lambda_{1}}
\end{gathered}
$$

In the same way:

$$
\begin{aligned}
& a_{i}=t_{0}=\frac{\alpha_{A} \cdot c_{i}-p_{A_{i}}-\left(\alpha_{A} \cdot c_{j}-p_{A_{j}}\right)+\lambda_{2}}{2 \lambda_{2}}= \\
& \frac{\alpha_{A}\left(2 c_{i}-1\right)-\left(p_{A_{i}}-p_{A_{j}}\right)+\lambda_{2}}{2 \lambda_{2}}
\end{aligned}
$$

Combined (2) and (3) can be obtained:

$$
\left\{\begin{array}{l}
c_{i}=\frac{1}{2}\left[1+\frac{\alpha_{C}\left(p_{A_{j}}-p_{A_{i}}\right)+\lambda_{2}\left(p_{C_{j}}-p_{C_{i}}\right)}{\left(\lambda_{1} \lambda_{2}-\alpha_{C} \alpha_{A}\right)}\right] \\
a_{i}=\frac{1}{2}\left[1+\frac{\alpha_{A}\left(p_{C_{j}}-p_{C_{i}}\right)+\lambda_{1}\left(p_{A_{j}}-p_{A_{i}}\right)}{\left(\lambda_{1} \lambda_{2}-\alpha_{C} \alpha_{A}\right)}\right]
\end{array}\right.
$$

$m_{C}$ and $m_{A}$ represent the marginal cost of new media platforms providing products or services to consumers and advertisers respectively.

The profit function of the platform i:

$$
\pi_{i}=\left(p_{C_{i}}-m_{C}\right) c_{i}+\left(p_{A_{i}}-m_{A}\right) a_{i}
$$

\section{B. Model Derivation}

The above (4) form (5) can be obtained:

$$
\begin{aligned}
\pi_{i}=\frac{1}{2}\left(p_{C_{i}}-m_{C}\right) & {\left[1+\frac{\alpha_{C}\left(p_{A_{j}}-p_{A_{i}}\right)+\lambda_{2}\left(p_{C_{j}}-p_{C_{i}}\right)}{\left(\lambda_{1} \lambda_{2}-\alpha_{C} \alpha_{A}\right)}\right] } \\
& +\frac{1}{2}\left(p_{A_{i}}-m_{A}\right)[1 \\
& \left.+\frac{\alpha_{A}\left(p_{C_{j}}-p_{C_{i}}\right)+\lambda_{1}\left(p_{A_{j}}-p_{A_{i}}\right)}{\left(\lambda_{1} \lambda_{2}-\alpha_{C} \alpha_{A}\right)}\right]
\end{aligned}
$$

The sufficient and necessary condition of $\pi_{i}$ for the existence of a maximum is: $4 \lambda_{1} \lambda_{2}>\left(\alpha_{C}+\alpha_{A}\right)^{2}$;

While $\frac{\partial \pi_{i}}{\partial p_{A_{i}}}=0 \Longrightarrow$

$$
\frac{1}{2}\left[1+\frac{\alpha_{A}\left(p_{C_{j}}-p_{C_{i}}\right)+\lambda_{1}\left(p_{A_{j}}-p_{A_{i}}\right)-\lambda_{1}\left(p_{A_{i}}-m_{A}\right)-\alpha_{C}\left(p_{C_{i}}-m_{C}\right)}{\left(\lambda_{1} \lambda_{2}-\alpha_{C} \alpha_{A}\right)}\right]=0
$$

Because the equilibrium conclusion of the Hotelling model is symmetric, Make:

$$
p_{C_{1}}=p_{C_{2}}=p_{C}, p_{A_{1}}=p_{A_{2}}=p_{A}, c_{1}=c_{2}=\frac{1}{2}, a_{1}=a_{2}=\frac{1}{2}
$$

And substitute it in the formula of profit maximization of the above platform operators:

$$
\left\{\begin{array}{l}
p_{C}=m_{C}+\lambda_{1}-\frac{\alpha_{A}}{\lambda_{2}}\left(\alpha_{C}+p_{A}-m_{A}\right) \\
p_{A}=m_{a}+\lambda_{2}-\frac{\alpha_{C}}{\lambda_{1}}\left(\alpha_{A}+p_{C}-m_{C}\right)
\end{array}\right.
$$


Solution:

$$
\left\{\begin{array}{l}
p_{C}=m_{C}+\lambda_{1}-\alpha_{A} \\
p_{A}=m_{a}+\lambda_{2}-\alpha_{C}
\end{array}\right.
$$

According to the (5) and model symmetry equilibrium conditions, the demand price elasticity of the consumer and the advertiser on the audio-visual content products displayed on the new media platform can be obtained: $\xi_{C}=\frac{p_{C}}{\lambda_{1}} ; \xi_{A}=\frac{p_{A}}{\lambda_{2}}$ then the Lerner exponent is:

$$
\left\{\begin{array}{l}
\frac{1}{\xi_{C}}=\frac{p_{C}-m_{C}+2 \alpha_{A} \cdot n_{A}}{p_{C}} \\
\frac{1}{\xi_{A}}=\frac{p_{A}-m_{A}+2 \alpha_{C} \cdot n_{C}}{p_{A}}
\end{array}\right.
$$

\section{Main Conclusion}

From the price structure formula of the (7) type of instant platform operators, it can be seen that when there are two new media platforms in the market to deal with audio-visual content products at the same time, that is, when there is a competitive relationship between platforms, the platform still needs to be deducted for the pricing of its two-sided users (consumers and advertisers). The cross network externalities on the other side are $\alpha_{C}$ and $\alpha_{A}$. In addition, the differentiation of products or services provided by the platform to users should also be considered between competing platforms, namely $\lambda_{1}$ and $\lambda_{2}$. In the platform competition under the single ownership of the two-sided users, the two platforms have the same fees, and the level of the charge for one user is proportional to the degree of the differentiation of the products or a service provided by the platform, and is inversely proportional to the users cross network externality. When the degree of differentiation of the competitive new media platform is low, the platform will be crossed to the cross network. Users with higher externalities are usually priced at lower prices or even free, that is, asymmetric pricing.

From the Lerner Index Formula of the two-sided consumer or advertisement on the (15) competitive new media platform, it can be seen that compared with the monopoly of the new media platform in the market, the Lerner Index gives double weight to the externality effect of the cross network between consumers and advertisers at this time. If $\mathrm{A}$, a new media platform operator in the market, improves its consumer price, the consumer will flow to another new media platform operator B in the market, and the consumer's cross network externality will lead to the platform operator A's advertisers to follow the consumer to the platform operator B. Therefore, the competition between the new media platforms leads to the double effects of the cross network externalities between the consumers and the advertisers, which further restricts the space for pricing and deployment of the platform operators.

\section{PRICING Strategy ANALYSIS BASED ON TWO-SIDED MARKET MODEL}

The asymmetric price structure used by the new media platform operators in the two-sided market is mainly caused by the cross network externalities between the two-sided consumers and the advertisers. The platform operator aims to attract consumers as much as possible through this pricing method, and then to attract advertisers from other side to the platform through the cross network externality. Because the negative cross network externalities brought by platform advertisers to consumers will obviously weaken the attraction of audio-visual content products and related services on the platform, the platform operators will put forward an extraordinarily high charge to advertisers to balance the cost and produce huge profits, and the consumers and advertisements. The internalization of cross network externalities is the core of platform operators' adoption of the price structure.

The pricing model of competitive new media platform reflects the greater the degree of differentiation of audio-visual content products, the less the price elasticity of the demand price for the visual and audio-visual content products displayed on the new media platform. However, the competition between the operators of the new media platform leads to the double effects of the cross network externalities between the consumers and the advertisers, which further limits the space for the platform operators to allocate the pricing. Therefore, based on the characteristics of the two-sided market, the pricing of the consumer side by the new media platform operators is mainly influenced by the differences in the audio-visual content products; while the pricing of the other advertisers is mainly influenced by the consumers' cross network externalities.

\section{ACKNOWLEDGMENT}

This paper is one of the stage achievements of the research and cultivation project of Communication University of China, which is based on the big data film box office research, and is funded by the special funds of the basic scientific research service of the Central University.

\section{REFERENCES}

[1] Guan Lixia. Electronic Commerce Research on audio-visual products [ J]. Beijing Broadcasting Institute, 2004, (03): 116-118

[2] Chen Fang. Research on pricing strategy of e-commerce B2B platform based on two sided market theory [D]. Zhejiang: Ningbo University, 2015.8-10.

[3] Quai Ling Bo. Video website pricing model based on two sided market theory [D]. Jiangsu: Soochow University, 2013.8-12.

[4] Ji Hanlin, pipe tin exhibition. Two-sided market and its pricing strategy research [J]. foreign economics and management, 2006, 28 (03): 35-41.

[5] Caillaud, B and B Jullien. Chicken \& egg: competition among intermediation service service Jullien. 2002, 